\title{
"Te das cuenta de que el mundo puede ser tan distinto y similar al mismo tiempo": Telecolaboración y desarrollo de la competencia intercultural en la educación superior
}

\author{
Ana SEVILla PAVÓN \\ Departament de Filologia Anglesa i Alemanya \\ Universitat de València \\ Ana.M.Sevilla@uv.es \\ Julia HABA OSCA \\ Departament de Filologia Anglesa i Alemanya \\ Universitat de València \\ Julia.Haba@uv.es
}

Recibido: 29 de febrero de 2016

Aceptado: 5 de septiembre de 2016

\begin{abstract}
RESUMEN
Este artículo versa sobre la integración de la telecolaboración, o intercambio intercultural en línea, en contextos de educación superior, para el desarrollo de la competencia intercultural en el aula inglés como lengua extranjera. Se analiza, desde la perspectiva de los estudiantes, un intercambio de telecolaboración en que participantes de España y de Estados Unidos colaboraron en grupos interculturales y plurinacionales para llevar a cabo una serie de tareas cuya complejidad cognitiva iba progresivamente en aumento: creación de un perfil digital, grabación de un vídeo de presentación, interacción multimodal (texto, audio, videoconferencia) para discutir y debatir los diferentes temas propuestos, creación de relatos digitales y realización de una presentación oral. Los datos cualitativos y cuantitativos analizados fueron obtenidos mediante un pre- y un post-cuestionario. La complejidad de llevar a cabo este tipo de proyectos apunta la necesidad de una revisión curricular que permita la "normalización" (Bax, 2000, 2003; Chambers y Bax, 2006) de las Tecnologías de la Información y de la Comunicación en el aula para un uso efectivo e integrado de las mismas en las prácticas docentes del siglo XXI. Asimismo, el apoyo institucional en forma de reconocimiento, provisión de recursos e inclusión de este tipo de proyectos como parte integral de las diferentes asignaturas se perfila como el elemento clave para que este tipo de iniciativas alcancen los objetivos propuestos.
\end{abstract}

Palabras clave: telecolaboración, competencia intercultural, educación superior, normalización 


\title{
"You realize that the world can be so different and similar at the same time": Telecollaboration and the development of the intercultural competence in higher education
}

\begin{abstract}
This paper revolves around the integration of telecollaboration, or online intercultural exchange, in higher education settings for the development of the intercultural competence in the English as a Foreign Language classroom. The perspective of the participants in a telecollaboration exchange from both Spain and the United States is analysed. The students collaborated in plurinational, multicultural groups so as to complete a set of tasks, the cognitive complexity of which was progressively increased: creating a digital profile, recording an introduction video, multimedia interaction (e.g. text, audio, videoconferencing) discussing and debating suggested topics, creating digital stories and planning and delivering an oral presentation. The qualitative and quantitative data analysed were gathered by means of a pre- and a post-questionnaire. The complexity of carrying out these kinds of projects points out the need for a curricular revision which allows for the normalisation (Bax, 2000, 2003; Chambers \& Bax, 2006) of Information and Communications Technologies in the classroom. This in turn would imply an effective and integrated use of those technologies within 21 st century teaching practices. Furthermore, institutional support in the form of recognition, provision of resources and inclusion of such projects in the syllabus stands out as the key element for the achievement of the goals of these kinds of initiatives.
\end{abstract}

Key words: telecollaboration, intercultural competence, higher education, normalization

\section{"Tu te rends compte que le monde peut être tellement divers et similaire en même temps": Télécollaboration et développement de la compétence interculturelle dans l'enseignement supérieur}

\section{RÉSUMÉ}

Cet article se concentre sur l'intégration de la télécollaboration, ou échange interculturel en ligne, dans le contexte de l'enseignement supérieur, pour le développement de la compétence interculturelle dans la classe d'anglais comme langue étrangère. On analyse, du point de vue des étudiants, l'échange de télécollaboration dans lequel les participants de l'Espagne et les États-Unis ont collaboré à des groupes interculturels et multinationaux pour mener à bien une série de tâches dont la complexité cognitive augmentait progressivement: création d'un profil digital, enregistrement d'une vidéo de présentation, interaction multimodale (texte, audio et vidéoconférence) pour discuter et débattre des thèmes proposés, création des histoires numériques et réalisation d'un exposé oral. Les données qualitatives et quantitatives analysées ont été recueillies par le biais d'un pré- et un post-questionnaire. La complexité de la réalisation de ce genre de projet souligne notamment la nécessité d'une révision des programmes d'enseignement permettant la «normalisation» (Bax, 2000, 2003; Chambers \& Bax, 2006) des Technologies de l'information et de la communication aux cours de langues étrangères pour l'utilisation efficace et intégrée de ces technologies dans les pratiques pédagogiques du XXIe siècle. En outre, l'appui institutionnel sous la forme de la reconnaissance, la provision de ressources et l'inclusion de ces projets en tant que partie intégrante de différents sujets apparaissent comme essentielles pour que ces initiatives puissent atteindre les objectifs proposés. 
Mots-clés: télécollaboration, compétence interculturelle, enseignement supérieur, normalisation

SUMARIO: 1. Introducción; 2. Marco Teórico; 2.1. La competencia intercultural en el aula de lengua extranjera; 2.2. La telecolaboración en la educación superior; 3. El proyecto "Telecolaboración Wofford-Valencia"; 4. Metodología; 4.1 Participantes; 4.2 Procedimiento; 5. Análisis de los resultados; 5.1 Competencia intercultural; 5.2 Actitudes hacia el proyecto; 5.3. Dificultades identificadas; 5.4 Volumen de trabajo; 6. Consideraciones finales; 7. Bibliografía

\section{INTRODUCCIÓN}

Este artículo se centra en la integración de la telecolaboración, o intercambio intercultural en línea, en el aula de inglés en contextos de educación superior, para el desarrollo de la competencia intercultural en el marco del aprendizaje del inglés como lengua extranjera. El objetivos principal es analizar percepciones de los estudiantes que participaron en dicho intercambio de telecolaboración, a partir de sus respuestas cualitativas y cuantitativas a las preguntas de respuesta abierta y cerrada a un pre- y un post-cuestionario. El intercambio se produjo en el primer semestre del curso académico 2015-2016, siendo las instituciones participantes una universidad de España y otra de Estados Unidos. Los estudiantes dedicaron alrededor de 2 horas semanales, durante un período de 14 semanas, a realizar tareas -cuya complejidad a nivel cognitivo iba en aumento de manera progresiva- en grupos interculturales de entre 4 y 5 alumnos. Dichas tareas, ordenadas según la Taxonomía de Bloom (Bloom, Engelhart, Furst y Krathwohl, 1956) y una de sus revisiones posteriores (Anderson y Krathwohl, 2001), fueron, en primer lugar, tareas iniciales se centraban en capacidades cognitivas y habilidades de pensamiento de orden inferior, que implicaban recordar, comprender e intercambiar información. Seguidamente, se trabajaron tareas de mayor complejidad cognitiva, pues los alumnos habían de aplicar una serie de conocimientos y analizar información y situaciones específicas para tratar de proporcionar soluciones. Por último, la tarea principal del proyecto implicó el desarrollo de habilidades cognitivas de orden superior, tales como la creación y la evaluación, tal y como se explica más adelante.

La importancia de iniciativas como esta, que impliquen un contacto directo del alumnado con los hablantes nativos de la lengua meta o L2, ha sido resaltada en numerosas ocasiones por la Comisión Europea, a través de sus recomendaciones en relación a la inclusión de una amplia variedad de aspectos no lingüísticos, tales como el conocimiento sociolingüístico, la consciencia intercultural y la capacidad de aprender a aprender (2001). Del mismo modo, existen numerosos ejemplos en la literatura del modo en que la telecolaboración favorece no solo la adquisición lingüística sino también el desarrollo de la competencia intercultural (Álvarez González, 2010; Bandura, 2001; Byram, 1997, 2000; Byram y Fleming, 1998), 
junto con competencias instrumentales, sistémicas e interpersonales (Pérez Cañado, 2010, 2013; Vinagre Laranjeira, 2014, 2016).

A pesar de que multitud de autores señalan los numerosos beneficios de este tipo de intercambios de telecolaboración, como son la exposición a input auténtico en la lengua meta y contacto directo con la cultura meta (Eck, Legenhausen y Wolff, 1995; Hauck y Youngs, 2008; Kern, Ware y Warschauer, 2004), la complejidad de llevar a cabo este tipo de proyectos -a nivel técnico, de planificación, de currículum y de apoyo institucional, entre otros- apunta la conveniencia de una revisión del currículum que facilite la "normalización" (Bax, 2000, 2003; Chambers y Bax, 2006) de las Tecnologías de la Información y de la Comunicación (TIC) en el aula, favoreciendo un uso efectivo e integrado de las mismas en las prácticas docentes del siglo XXI y, más específicamente, en la didáctica de la lengua extranjera. Del mismo modo, el apoyo institucional en forma de reconocimiento, provisión de recursos e inclusión de este tipo de proyectos como parte integral de las diferentes asignaturas se perfila como elemento clave para el éxito de este tipo de iniciativas.

\section{MARCO TEÓRICO}

\subsection{La competencia intercultural en el aula de lengua extranjera}

Numerosas publicaciones y una miríada de autores han tratado de definir la competencia intercultural y han señalado su importancia en el aula de lenguas extranjeras, sin que exista un consenso en torno a dicha definición. Guilherme (2004, 297-300) define la competencia intercultural como la habilidad para interactuar de manera efectiva con personas de otras culturas que nosotros reconocemos como diferente de la nuestra. De acuerdo con Brislin y Yoshida (1994), los aspectos culturales que comparten las culturas en contacto en el proceso comunicativo son denominados culture-general y los aspectos en los cuales difieren, culture-specific. Por lo tanto, de aquí se deduce que cuanto menos aspectos del tipo culture-general compartan las culturas y más aspectos del tipo culturespecific se encuentren, más diferentes resultarán ambas culturas.

Según Byram (1997), la competencia intercultural es más compleja que la comunicativa, debido a que esta última se centra, sobre todo, en establecer y en mantener las relaciones, en vez de ser un mero intercambio de mensajes e información. Por su parte, Meyer (1990) incluye entre las habilidades que tiene que desarrollar el alumnado de lengua y cultura extranjera la adaptabilidad y flexibilidad. Guilherme (2004) apunta que la interacción intercultural se lleva a cabo generalmente en la lengua nativa de alguno de los participantes en la comunicación. En consecuencia, la competencia intercultural puede entenderse como la habilidad para interactuar en la propia lengua con personas de otro país y de otra cultura (Byram y Fleming, 1998). Dicho de otro modo, la competencia intercultural sería considerada la capacidad para comunicarse en una lengua extranjera de manera efectiva, con lo que no bastaría con hablar la lengua meta o L2 
de manera fluida, sino que sería necesario también saber comunicarse con personas de procedencias lingüísticas y culturales diversas. De este modo, se evitaría el riesgo de malentendidos que advierten autores como Bennett, Bennett y Allen (2003, 237), pues "the person who learns language without learning culture risks becoming a fluent fool".

Por otro lado $\mathrm{y}$, en cuanto al encuentro donde tiene lugar el intercambio comunicativo intercultural, en él se produce, de manera acompasada, una interacción entre las diferentes identidades sociales, sus percepciones acerca de las otras identidades y, quizás, la toma de conciencia de la identidad dominante según las circunstancias particulares que se estén dando (Byram y Fleming, 1998). Habida cuenta de la complejidad de la dimensión cultural y su impacto en los intercambios comunicativos, es importante señalar el nuevo papel que cobra la adquisición de la competencia intercultural por parte del alumnado. Así, una adecuada integración de la teoría en la práctica docente habría de traducirse en la conveniente interpretación, reflexión, explicación y praxis en lo que se refiere a la formación del profesorado, para que los docentes dispongan de herramientas que les permitan promover la adquisición de esta competencia. En relación a lo anterior, Pastor (2005) afirma que la figura del docente de lenguas y culturas extranjeras ya no es aquel embajador de la lengua y la cultura extranjera, desde una visión simplista, estandarizada y estática de la lengua y la cultura. De este modo, actualmente, entre las competencias y destrezas del docente de lenguas extranjeras del siglo XXI se incluye el conocimiento y capacidad de transmisión de la naturaleza interactiva del aprendizaje de las lenguas y las culturas, junto con sus implicaciones sociales, políticas y éticas. Para ello, el profesorado no debe ser educado desde la "aculturalización" en la nueva cultura, sino que es importante que se le forme desde un espíritu crítico, hacia la enseñanza-aprendizaje de las lenguas y culturas extranjeras, lo cual conlleva también reflexionar acerca de las suyas, así como sobre la interacción intercultural.

\subsection{La telecolaboración en la educación superior}

Un creciente y cada vez más robusto conjunto de investigaciones en torno a la telecolaboración, también llamada intercambio intercultural en línea, movilidad virtual, interacción e intercambio en línea (Dooly y O'Dowd, 2012), o e-tandem, unido a un número cada vez mayor de plataformas de telecolaboración, demuestran que este tipo de acciones educativas se consideran especialmente relevantes en el contexto actual. Entre las plataformas en que pueden realizarse intercambios de telecolaboración, destacan: Unicollaboration, creada en el marco del proyecto europeo INTENT, especialmente dedicada a los intercambios entre universidades; ETwinning, una plataforma creada en el seno de la Unión Europea y dedicada principalmente a los intercambios entre escuelas de educación primaria y secundaria; y también Epals, una plataforma creada por una empresa privada. En cuanto a los proyectos que plantean diversos modelos $\mathrm{e}$ iniciativas de 
telecolaboración, cabe señalar: el proyecto TeCola, Pedagogical differentiation through telecollaboratlon and gaming for intercultural and content integrated language teaching, el proyecto NIFLAR, Networked Interaction in Foreign Language Acquisition and Research; TILA, Telecollaboration for Intercultural Language Acquisition; el anteriormente mencionado proyecto INTENT, Integrating Telecollaborative Networks into Foreign Language Higher Education; CULTURA; Soliya Connect Program; y Teletandem, entre otros. La amplia variedad de términos que diferentes investigadores y docentes han venido empleando en los últimos años para referirse a la telecolaboración ha venido acompañada, además, de un sinfín de definiciones del concepto, lo cual pone de manifiesto, una vez más, el interés que esta práctica pedagógica ha venido suscitando en el aula de idiomas. A pesar de que esta vasta cantidad de términos y definiciones puede resultar abrumadora, las diferentes maneras en que se ha entendido y explicado coinciden en ciertos elementos clave, a saber:

- que se trata de una metodología docente que asume una visión socioconstructivista del aprendizaje (Vygotsky, 1978), centrada en el alumno (Jones, 2007; Vygotsky, 1978) y basada en tareas (Richards y Rodgers, 2014; Skehan, 2003a, 2003b; Willis, 1996);

- que es una forma de movilidad virtual empleada de manera creciente en diferentes niveles educativos, incluyendo la educación superior, sustituyendo y complementando la movilidad física de los estudiantes (O'Dowd, 2013), pues permite un contacto de primera mano con la lengua y cultura metas (Eck, Legenhausen y Wolf, 1995; Hauck y Youngs, 2008; Kern, Ware y Warschauer, 2004);

- que se caracteriza por la colaboración e interacción entre alumnos en diferentes lugares del mundo que asisten a clases de idiomas paralelas y utilizan la comunicación a través de internet o comunicación mediada por ordenador síncrona y asíncrona. Dicha comunicación se produce a través de herramientas como los entornos virtuales de aprendizaje, el correo electrónico, el chat de discusión síncrona, los foros de discusión asíncrona, la videoconferencia, etc. para la interacción social, el diálogo, el debate y el intercambio intercultural (Belz, 2003);

- que el intercambio puede producirse entre hablantes no nativos de una misma lengua meta, en cuyo caso se consideraría un intercambio de L2; o bien producirse entre hablantes nativos de una lengua cuya lengua meta o L2 es la lengua de sus respectivos compañeros, en cuyo caso se considera un intercambio de tipo L1 o primera lengua, como el presentado en este artículo. 


\section{EL PROYECTO "TELECOLABORACIÓN WOFFORD-VALENCIA"}

El proyecto "Telecolaboración Wofford-Valencia" es una iniciativa que cuenta con el apoyo institucional del Servicio de Formación permanente e Innovación educativa de la Universitat de València que se llevó a cabo entre estudiantes de la Universitat de València, España; y del Wofford College, Carolina del Sur, Estados Unidos. En el caso de los 32 participantes de la Universidad de València, se trataba de estudiantes de primer curso del Grado en Negocios Internacionales, que estaban cursando la asignatura troncal de 6 créditos ECTS (60 horas lectivas) de "Inglés para los Negocios III", con un nivel de inglés que oscilaba entre el B2 y el C1 del Marco Común Europeo de Referencia para las Lenguas (MCERL, Consejo de Europa, 2001). Los estudiantes tuvieron 4 horas de clases presenciales a la semana durante un período de 14 semanas, de septiembre a diciembre de 2015, esto es, durante el primer cuatrimestre del curso 2015-2016. De esas 4 horas semanales, entre 1 y 2 horas se dedicaban a tareas relacionadas con el proyecto.

Los objetivos principales del proyecto eran el desarrollo de destrezas lingüísticas y comunicativas de los estudiantes, así como el desarrollo de la competencia intercultural. Entre los objetivos secundarios de proyecto se incluían el desarrollo de la autonomía en el aprendizaje (Little, 1997), el pensamiento crítico, la creatividad y el desarrollo de la competencia digital (Knobel y Lankshear, 2008; Martin y Madigan, 2006). Las características propias de la asignatura de "Inglés para los Negocios III" facilitaron la realización exitosa del proyecto ya que, como se recoge en la guía docente, de las 4 horas semanales de clases presenciales, una hora se dedica a actividades de tipo teórico, otra hora está destinada a la realización de actividades prácticas y las dos horas restantes tienen un carácter eminentemente práctico y comunicativo. Las dos horas de laboratorio anteriormente mencionadas se imparten en laboratorios informáticos que implican un uso integrado de las TIC, facilitando la tan ansiada "normalización" a que aspiraban educadores e investigadores como Bax $(2000,2003)$ y Chambers y Bax (2006). A continuación, se resume el cronograma de actividades y tareas llevadas a cabo en el marco del proyecto, así como los modos y herramientas de comunicación empleadas en cada una de las fases del mismo:

\begin{tabular}{|l|l|l|}
\hline \multicolumn{5}{|c|}{ FASE 1: PRE-INTERCAMBIO / PREPARACIÓN } \\
\hline Tema & \multicolumn{1}{|c|}{ Tareas } & \multicolumn{1}{|c|}{$\begin{array}{l}\text { Modo y Herramientas } \\
\text { de Comunicación }\end{array}$} \\
\hline $\begin{array}{l}\text { Empezamos a } \\
\text { conocernos y } \\
\text { a romper el } \\
\text { hielo }\end{array}$ & $\begin{array}{l}\text { 1. Creación del perfil digital } \\
\text { 2. Pre-cuestionario sobre la tele- } \\
\text { colaboración }\end{array}$ & $\begin{array}{l}\text { Asíncrona: } \\
\text { imágenes, grabación de } \\
\text { vídeo e hipervínculos en } \\
\text { Google+ }\end{array}$ \\
$\begin{array}{l}\text { 4. Formular preguntas para romper el hielo } \\
\text { 5. Grabación de un vídeo breve de } \\
\text { presentación }\end{array}$ & \\
\hline
\end{tabular}




\begin{tabular}{|c|c|c|}
\hline \multicolumn{3}{|c|}{ FASE 2: INTERCAMBIO (1) } \\
\hline Tema & Tareas & $\begin{array}{l}\text { Modo y Herramientas } \\
\text { de Comunicación }\end{array}$ \\
\hline $\begin{array}{l}\text { Desafiamos } \\
\text { los } \\
\text { estereotipos }\end{array}$ & $\begin{array}{l}\text { 1. Formación de grupos } \\
\text { 2. Redacción de una historia sobre el uso } \\
\text { de la lengua } \\
\text { 3. Visualización del vídeo TED de } \\
\text { Chimamanda Ngozi Adichie "The danger } \\
\text { of a single story" e intercambio de } \\
\text { comentarios en el foro } \\
\text { 4. Discusión sobre los estereotipos de } \\
\text { diferentes países }\end{array}$ & $\begin{array}{l}\text { Asíncrona: comentarios } \\
\text { en formato de texto en el } \\
\text { foro de Google+ } \\
\text { Síncrona: conversación } \\
\text { por videoconferencia a } \\
\text { través de la herramienta } \\
\text { Hangouts }\end{array}$ \\
\hline \multicolumn{3}{|c|}{ FASE 3: INTERCAMBIO (2) } \\
\hline Tema & Tareas & $\begin{array}{l}\text { Modo y Herramientas } \\
\text { de Comunicación }\end{array}$ \\
\hline $\begin{array}{l}\text { Globalización } \\
\text { y } \\
\text { comunicación } \\
\text { intercultural }\end{array}$ & $\begin{array}{l}\text { 1.Hacer una lista de los } 5 \text { "mejores países" } \\
\text { 2. Visualización del vídeo TED de Simon } \\
\text { Anholt: "Which country does the most } \\
\text { good for the world?" } \\
\text { 3. Discusión en grupo y propuesta de } \\
\text { posibles soluciones a estudios de caso } \\
\text { sobre comunicación intercultural }\end{array}$ & $\begin{array}{l}\text { Asíncrona: comentarios } \\
\text { en formato de texto en el } \\
\text { foro de Google+ } \\
\text { Síncrona: conversación } \\
\text { por videoconferencia a } \\
\text { través de la herramienta } \\
\text { Hangouts, edición } \\
\text { simultánea de un } \\
\text { documento de texto para } \\
\text { realizar la propuesta de } \\
\text { solución de los estudios } \\
\text { de caso }\end{array}$ \\
\hline \multicolumn{3}{|c|}{ FASE 4: INTERCAMBIO (3) } \\
\hline Tema & Tareas & $\begin{array}{l}\text { Modo y Herramientas } \\
\text { de Comunicación }\end{array}$ \\
\hline $\begin{array}{l}\text { Un producto } \\
\text { innovador y } \\
\text { revolucionario }\end{array}$ & $\begin{array}{l}\text { 1. Creación de relatos digitales sobre el } \\
\text { producto innovador y revolucionario } \\
\text { 2. Presentaciones orales ante los inversores } \\
\text { en la Feria de negocios } \\
\text { 3. Redacción de un informe grupal sobre } \\
\text { la decisión de los inversores } \\
\text { 4. Evaluación por pares e intercambio de } \\
\text { feedback y comentarios a través de las } \\
\text { fichas de evaluación y en el foro de } \\
\text { discusión en línea } \\
\text { 5. Discusión con los asesores extranjeros } \\
\text { en cuando a la adaptación del producto } \\
\text { innovador a los mercados de los } \\
\text { respectivos países }\end{array}$ & $\begin{array}{l}\text { Asíncrona: } \\
\text { presentaciones orales en } \\
\text { grupos locales, } \\
\text { comentarios en formato } \\
\text { de texto en el foro de } \\
\text { Google+, redacción } \\
\text { asíncrona del guión en } \\
\text { GoogleDocs, redacción } \\
\text { del informe de los } \\
\text { inversores, fichas de } \\
\text { evaluación de las } \\
\text { presentaciones orales y } \\
\text { de los relatos digitales }\end{array}$ \\
\hline
\end{tabular}




\begin{tabular}{|c|c|c|}
\hline & & $\begin{array}{l}\text { Síncrona: conversación } \\
\text { por videoconferencia con } \\
\text { los asesores a través de la } \\
\text { herramienta Hangouts }\end{array}$ \\
\hline \multicolumn{3}{|c|}{ FASE 5: POST-INTERCAMBIO / EVALUACIÓN } \\
\hline Tema & Tareas & $\begin{array}{l}\text { Modo y Herramientas } \\
\text { de Comunicación }\end{array}$ \\
\hline $\begin{array}{l}\text { Evaluamos el } \\
\text { proyecto y nos } \\
\text { despedimos }\end{array}$ & $\begin{array}{l}\text { 1. Post-cuestionario sobre telecolaboración } \\
\text { 2. Entrevistas a grupos focales } \\
\text { 3. Realización de pruebas finales en los } \\
\text { respectivos } \\
\text { 4. Grabación de vídeos colectivos finales e } \\
\text { intercambio de comentarios de despedida }\end{array}$ & $\begin{array}{l}\text { Asíncrona: Grabación de } \\
\text { vídeos colectivos finales } \\
\text { a modo de despedida e } \\
\text { intercambio de } \\
\text { comentarios en formato } \\
\text { de texto en el foro de } \\
\text { Google+ }\end{array}$ \\
\hline
\end{tabular}

Tabla 1. Fases del intercambio de telecolaboración. Fuente: elaboración propia

Tal y como puede apreciarse en el cronograma anterior, las tareas realizadas durante el proyecto fueron ordenadas y repartidas a lo largo del semestre de acuerdo con el nivel de dificultad cognitiva y de habilidades de pensamiento de cada una de ellas (Anderson y Krathwohl, 2001; Bloom, Engelhart, Furst, Hill y Krathwohl, 1956), con el fin de garantizar que dicho nivel fuera aumentando de manera progresiva.

Dichas tareas fueron, en primer lugar, tareas iniciales que fomentaban capacidades cognitivas y habilidades de pensamiento de orden inferior, las cuales implicaban recordar, comprender e intercambiar información. Así, los estudiantes crearon su perfil digital en la plataforma Google+; rellenaron un pre-cuestionario y realizaron una prueba de diagnóstico que permitiera determinar su nivel de partida; formularon una serie de preguntas para comenzar a conocer a sus compañeros de intercambio y, así, romper el hielo; y grabaron un vídeo de presentación de alrededor de un minuto de duración, en sus lenguas metas respectivas.

Seguidamente, en las fases sucesivas del proyecto se trabajaron tareas de mayor complejidad cognitiva, pues los alumnos habían de aplicar una serie de conocimientos y analizar información y situaciones específicas para tratar de proporcionar soluciones. Así, los estudiantes compartieron y analizaron críticamente historias y recuerdos en relación a experiencias personales previas (tanto positivas como negativas) relacionadas con el uso de la lengua meta; analizaron un vídeo sobre la importancia de tener en cuenta la multidimensionalidad de la comunicación y comprensión intercultural; y discutieron los estereotipos de sus respectivos países. A continuación, debatieron y llegaron a acuerdos respecto a qué países hacían las contribuciones más positivas al mundo; y plantearon posibles soluciones a situaciones, en forma de estudios de caso, en que se habían producido malos entendidos desde el punto de vista intercultural. 
Por último, la tarea principal del proyecto, que se produjo en las últimas fases del mismo, implicó el desarrollo de habilidades cognitivas de orden superior, tales como la creación y la evaluación, en relación a la creación, presentación y evaluación de un producto innovador. Así, los estudiantes tuvieron que comunicarse y tomar decisiones en cuanto a la creación multimodal de relatos digitales sobre dicho producto innovador. Seguidamente, prepararon e hicieron presentaciones orales multimodales en una feria de negocios internacional con el fin de conseguir en apoyo de los inversores en el desarrollo y comercialización de su producto. A continuación, redactaron un informe grupal sobre su decisión, como inversores, en cuanto a la cantidad económica que invertirían en cada uno de los productos propuestos por sus compañeros. Por último, procedieron a la evaluación por pares y al intercambio de feedback y comentarios a través de unas fichas de evaluación que se les proporcionaron, así como mediante los comentarios en el foro de discusión en línea de Google+. El foro también se utilizó para discutir con los asesores extranjeros (sus compañeros de telecolaboración) diferentes propuestas para la adaptación del producto innovador a los mercados de los respectivos países.

\section{METODOLOGÍA}

Con el fin de analizar la opinión vertida por los participantes en el proyecto de telecolaboración, se llevó a cabo una encuesta, por ser el método más generalizado de observación cuantitativa (Giddens, 2014).

Se confeccionó una encuesta en línea con un total de 60 preguntas de índole mixta con la herramienta GoogleForms, en que las preguntas 1 a 45 eran cerradas, es decir, tenían un número fijo de posibles respuestas (siendo el valor 1 "muy en desacuerdo" y el valor 7 "muy de acuerdo") para facilitar los contrastes y comparaciones. Por su parte, las preguntas 46 a 60 eran abiertas, para permitir a los encuestados expresar sus ideas con mayor libertad y usando sus propias palabras, ampliando, así, el número de posibles categorías emergentes.

\subsection{Participantes}

Los datos cualitativos y cuantitativos analizados en el presente artículo fueron recopilados de las respuestas de 47 estudiantes $(\mathrm{N}=47)$ de ambas instituciones que participaron en el estudio, siendo el $70,2 \%$ mujeres y el $29,8 \%$ hombres. De entre estos participantes que rellenaron el post-cuestionario sobre el proyecto, 32 eran estudiantes de la UV que estaban cursando la asignatura troncal de 6 créditos ECTS "Inglés para los Negocios III", cuyo nivel oscilaba entre el B2 o intermedio alto y el C1 o avanzado del Marco Común Europeo de Referencia para las Lenguas (MCERL, Consejo de Europa, 2001); mientras que los 15 restantes eran estudiantes de diferentes grados, matriculados en un curso de español de nivel B1 o intermedio del MCERL ofrecido por Wofford College, Carolina del Sur, Estados Unidos. 


\subsection{Procedimiento}

Los participantes en el proyecto se dividieron en grupos de entre 4 y 5 estudiantes, habiendo en cada grupo al menos 2 estudiantes procedentes de cada uno de los dos países participantes. Se creó una comunidad de Google+ para que los estudiantes pudieran interactuar y así conocerse y organizarse de cara a la realización de las diferentes tareas propuestas semanalmente a lo largo de las 14 semanas de duración del proyecto.

Con el fin de recopilar información relativa a las opiniones y percepciones de los estudiantes en cuanto a diferentes aspectos del proyecto, incluida su contribución al desarrollo de la competencia intercultural en los estudiantes, se les pidió que rellenaran un post-cuestionario en línea a través de GoogleForms y, como se ha señalado anteriormente, se obtuvieron 47 respuestas al mismo.

Dicho post-cuestionario se dividía en cinco secciones: la sección A, "Demografía", que incluía las preguntas 1 a 7; la sección B, "Aprendizaje de lenguas extranjeras", que contenía las preguntas 8 a 17 ; la sección C, "Telecolaboración", con las preguntas 18 a 25; la sección D, "Competencia intercultural", en que figuraban las preguntas 26 a 45; y la sección E, "Aprendizaje de lenguas en un contexto intercultural", que constaba de las preguntas 46 a 60 . En cuanto al tipo de preguntas contenidas en cada una de las secciones, como se ha explicado anteriormente, 4 eran de preguntas de respuesta cerrada (secciones A a D), basadas en una escala Likert de 7 puntos, mientras que la última, sección E, consistía en preguntas de respuesta abierta.

\section{ANÁLISIS DE LOS RESULTADOS}

\subsection{Competencia intercultural}

El análisis, tanto cuantitativo como cualitativo, de las respuestas de los estudiantes permitió recabar información en cuanto a las percepciones, opiniones y sugerencias de los mismos en relación al proyecto. De entre las respuestas de los estudiantes cabe señalar, en primer lugar, aquellas relacionadas con el grado de contribución del proyecto al desarrollo de la competencia intercultural, desde la perspectiva de los propios estudiantes. Entre dichas respuestas se incluían:

- el acercamiento a otras cultura;

- la posibilidad de conocer a estudiantes de otros países y de comunicarse con ellos, superando las barreras espacio-temporales;

- las reflexiones en cuanto al modo en que el sesgo cultural puede influir en la manera de pensar de los individuos;

- la apreciación de la riqueza intercultural y del aprendizaje sobre otras culturas;

- la relación y facilidad de comunicación con personas de otras etnias o culturas; 
- la participación en actividades que impliquen el contacto intercultural;

- la preferencia en cuanto a elementos de la propia cultura o de otras;

-los aspectos más beneficiosos de trabajar en un entorno diverso y multicultural;

- el nivel de interés en conocer otras culturas;

- las opiniones específicas en relación a la comunicación intercultural a través de la telecolaboración.

Además, el análisis de las respuestas de los participantes permitió arrojar luz en relación a otros aspectos del proyecto, como:

- la motivación de cara al aprendizaje de lenguas;

- el grado de satisfacción respecto a la experiencia de trabajar con estudiantes de otros países en sus clases de idiomas y, específicamente, de hacerlo a través de la telecolaboración;

-las preferencias en cuanto a la modalidad de aprendizaje (en grupo o individualmente);

- las percepciones de mejora del nivel lingüístico;

- el desarrollo de la competencia digital;

- los aspectos más difíciles en relación al proyecto: lingüísticos, de organización temporal y estructural, y aquellos relacionados con la interdependencia positiva y los niveles dispares de compromiso entre los participantes;

- la valoración global del mismo, incluyendo sugerencias de mejora de cara a la realización de futuros intercambios de la misma índole.

Como adelantábamos, las respuestas de los participantes tras haber concluido el proyecto permitieron, en primer lugar, analizar las percepciones de los estudiantes respecto al grado de consecución de uno de los principales objetivos del proyecto de telecolaboración: el fomento de la competencia intercultural en los estudiantes. El hecho de que el $53 \%$ de los encuestados fueran de la opinión de que el proyecto fue una buena manera de ayudarles a desarrollar su competencia intercultural apunta a una consecución parcial de este objetivo (en alrededor de la mitad de los encuestados). Además, en la pregunta relacionada con el acercamiento a otras culturas, las respuestas muestran que el 55,2\% de los estudiantes consideró que la telecolaboración fue efectiva en relación a dicho acercamiento intercultural. Los porcentajes de nivel de acuerdo aumentaron en las preguntas siguientes, puesto que el $63,9 \%$ declaró ser consciente del modo en que su cultura afecta y condiciona su manera de pensar y el $61,7 \%$ se mostró muy de acuerdo con la afirmación sobre la necesidad de apreciar la riqueza intercultural y el aprendizaje sobre otras culturas, eligiendo los encuestados en dicha pregunta los valores máximos de la escala. 
Las respuestas anteriormente mencionadas pueden ser complementadas con las respuestas abiertas de los estudiantes a la pregunta sobre los aspectos más beneficiosos de trabajar en un entorno diverso y multicultural, en que la gran mayoría de los alumnos destacó un considerable número de aspectos positivos. En dichas afirmaciones, además, se aprecian categorías temáticas emergentes como la globalización, la superación de las barreras espacio-temporales y la capacitación profesional:

Respuesta 1 (R1): "It helps us because in the future we will be able to work better with people from other cultures".

R2: "Well, it distinguishes you from other people who only speak one language, and in a globalized world it's always good to know different languages and cultures because we interact all over the world without any barriers".

Entre las respuestas a las preguntas relacionadas con el contacto con otras culturas y etnias, encontramos, en primer lugar, que el círculo de amistades de la mayoría de los encuestados estaba formado por individuos pertenecientes a su misma cultura o grupo étnico, escogiendo el $63,7 \%$ de los valores máximos de la escala. Además, a la pregunta sobre su interés en participar en actividades que propiciaran el encuentro e interacción con personas de diferentes nacionalidades y grupos étnicos, el 57,4\% afirmaron estar de acuerdo o muy de acuerdo, demostrando, así, interés por conocer personas de orígenes diferentes a los propios.

En relación a si elegirían solo elementos de su propia cultura en caso de tener la oportunidad de diseñar una nueva cultura en un nuevo país (estando dicha pregunta relacionada con las tareas de la fase 3 del proyecto, sobre la globalización y la comunicación intercultural), el $57,4 \%$ se mostraron totalmente en desacuerdo, lo cual señala una apreciación de los elementos culturales y de las aportaciones positivas de otras culturas. Del mismo modo, ante la pregunta sobre la apreciación de la riqueza intercultural y del aprendizaje de otras culturas, el $61,7 \%$ del alumnado dijo estar muy de acuerdo, eligiendo el valor máximo dicho porcentaje de los encuestados. Además, la experiencia de conocer personas de otras culturas les resultaba especialmente estimulante al $89,3 \%$ de los encuestados, apuntando de nuevo al gran interés mostrado por los participantes ante la diversidad.

\subsection{Actitudes hacia el proyecto}

Del análisis de datos también se desprende que más de la mitad de los estudiantes se mostraron motivados o muy motivados en cuanto al aprendizaje de lenguas en sus respectivas instituciones: $72,3 \%$ de los estudiantes. Además, la mayoría de los estudiantes $(70,2 \%)$ se mostraron satisfechos con la experiencia de trabajar con estudiantes extranjeros en sus clases de idiomas, como demuestra su alto nivel de acuerdo con la afirmación de que les gustaría repetirla. No obstante, este número se redujo a $51,1 \%$ en el caso de realizar dicha experiencia intercultural a través de un proyecto de telecolaboración. 
A pesar de que los porcentajes señalados anteriormente, procedentes de la primera parte de la encuesta, no reflejan unos resultados óptimos, cabe destacar que, en la segunda parte de la encuesta (preguntas de respuesta abierta), una gran parte de los encuestados apuntó una serie de aspectos positivos adicionales relacionados con la telecolaboración. Dichos beneficios pueden enmarcarse dentro de diferentes categorías, como son: i) las actitudes positivas hacia el proyecto; ii) la utilidad del proyecto en cuanto al desarrollo de destrezas lingüísticas y comunicativas; iii) la originalidad y el poder motivador del proyecto; y iv) el entendimiento intercultural.

i) Actitudes positivas hacia el proyecto:

R1: "Very interesting, would like to try some more of telecollaboration". R2: "It was a unique and educational experience that I would not have been able to experience without this class".

ii) Desarrollo de habilidades lingüísticas y comunicativas: R3: "It has been very useful to improve our language skills".

R4: "Authenticity; I think there is a more direct emphasis and goal of communication rather than receiving a certain grade".

iii) Originalidad y poder motivador del proyecto:

R5: "A benefit was to do something different from traditional university tasks".

R6: "It was a great idea to get into communication with students that are almost the same age as we are and live in a totally different country because when you talk to them you realize that the world can be so different and similar at the same time".

iv) Entendimiento intercultural:

R7: "They allow people to experience and interact with people other cultures, giving them authentic knowledge of the cultures".

R8: "I would like to say that at the beginning I was quite worried because I didn't know anything about them but soon I realized that they were kind people like us".

R9: "Overall I think that my partner and I had things in common that made me realize that even though we are from totally different cultures there will always be something that connects us all".

R10: "Finally, apart from the jokes and the laughs, we shared the same thoughts about how we should all cooperate as a family to resolve our current 'differences and similarities' and as a result move forward in life whilst developing ourselves as persons and as a whole race”. 


\subsection{Dificultades identificadas}

Tras analizar las respuestas más destacadas sobre los aspectos de mayor complejidad del proyecto, según las percepciones de los participantes, estas se agruparon en diferentes categorías basadas en el tipo de dificultades que los estudiantes destacaron en sus respuestas: i) lingüísticas; ii) de volumen de trabajo y organización temporal; iii) de organización estructural y del trabajo; iv) de comunicación intercultural; $y, v)$ de interdependencia positiva.

i) Dificultades lingüísticas:

R1: "[there were] difficulties in understanding".

R2: "Sometimes it is really hard to communicate if you don't know a word because English is not your mother tongue".

ii) Dificultades en cuanto a la organización temporal:

R3: "Too busy schedules to incorporate it on our own".

R4: "It was helpful, but took a lot more time than was intended".

R5: "It was quite a nice and interesting experience, but it was timeconsuming,

too".

iii) Dificultades de organización estructural y del trabajo:

R6: "Agreement on the same topic".

R7: "To make decisions from different points of view".

R8: "Different working approaches (tasks need to be explained clearly)".

R9: "Great idea but not structured enough to be effective". R10: "It's a really nice idea but I think there is room for improvement. A good thing would be that all the students do same tasks at the same time".

iv) Dificultades de Comunicación Intercultural:

R11: "Remembering that everyone is not like you".

R12: "Overcoming subconscious biases".

R13: "I think the most challenging aspects are getting everyone to have an open mind to exploring cultures they are not familiar with".

R14: "To understand that people from different ethnicities react differently on the same conflict".

R15: "It is difficult to try and blend different cultural norms, etc., especially with communication".

R16: "It's hard to fully comprehend another culture because you don't find it normal. The most challenging part is understanding the purpose for something or the logic behind culture". R17: "It is important to be able to approach a conversation with no bias, or at least be able to be aware of your biases in order to be able to get the most out of a diverse, multicultural setting". 
R18: "It's probably the first moment when you get in touch with different cultural people. At first view, it can be hard because you might be shy, but you don't have to feel like that because it's the same for the other person".

v) Dificultades de interdependencia positiva:

R19: "It is semi-useful, but if you aren't matched with a partner who will respond on time it will be frustrating and pointless". R20: "I think it is a good idea and something that can really help students learn a lot about the culture while improving in the language. However, I do not think we used it very often".

La categoría iv), relacionada con las "Dificultades de Comunicación Intercultural", merece especial atención por el gran número de comentarios suscitados y por su relación con uno de los objetivos principales del proyecto. La percepción de dichas dificultadas y el nivel de profundidad de los comentarios recibidos en torno a esta cuestión tiene su reflejo en las interacciones de los estudiantes. Así, los comentarios de las respuestas 11 a 18 muestran una actitud de esfuerzo consciente por ser más abiertos y tolerantes, superar posibles prejuicios y estereotipos, conocer y comprender una cultura con la que no están familiarizados, entender posibles reacciones diversas ante un conflicto o normas de comunicación diferentes, entender la lógica de las diferentes normas, etc. La manifestación de dicha preocupación y esfuerzo consciente de los estudiantes queda patente en sus interacciones, como se aprecia en este ejemplo de discusión en torno a los estereotipos en que los estudiantes intentan encontrar puntos en común, al tiempo que matizan ciertos estereotipos:

Spanish student: "One stereotypes about American people is that they eat a lot of junk food following an unhealthy diet. Is it true? It is said that the Mediterranean diet (and Spanish diet too) is one of the best, I have to say that it depends a lot on the place you were eating (...)".

American Student: "Hey (Spanish student's name). I agree with your opinion on stereotypes. They are not always true but they can be in certain cases. For example, the stereotype you mentioned about Americans following an unhealthy diet and eating a lot of junk food is definitely true for college students. At least from my perspective I think that a lot of college students eat very unhealthy. We live off of fast food and microwave products. However, I know plenty of people who live very healthy lives and always watch what they eat".

Spanish student: "In Spain the same happens among college students. Junk food is cheaper and in my opinion, it is quite addictive too. However, as you have said, there are plenty of people who follow a healthy diet and also practice a lot of sports (...)".

De las afirmaciones anteriores y de las respuestas a la pregunta relacionada con la valoración global del proyecto se desprende que, como se ha expuesto 
anteriormente, los estudiantes, por un lado, manifestaron actitudes positivas hacia el proyecto y al modo en que este contribuyó al desarrollo de la competencia intercultural. En este sentido, cabe destacar la valoración positiva de aspectos tales como los beneficios del trabajo en un entorno diverso y multicultural que les prepara para desenvolverse con éxito en posibles entornos laborales futuros. Ello se percibe como especialmente relevante en su formación y preparación para el contexto actual de globalización en que impera la necesidad de superación de las barreras espacio-temporales, culturales y lingüísticas. Otros de los aspectos del proyecto que fueron valorados positivamente por los estudiantes fueron: la contribución positiva del proyecto al desarrollo de habilidades lingüísticas y comunicativas; la originalidad y poder motivador de la propuesta; el contacto directo con la lengua y la cultura metas; y el modo en el que el proyecto favoreció el entendimiento intercultural.

No obstante, los estudiantes también aportaron críticas. La primera de las críticas tuvo que ver con dificultades lingüísticas y de comunicación, tanto a nivel de uso de la lengua como en relación a las diferencias culturales que, según la opinión de algunos alumnos, dificultaban la realización exitosa de las diferentes tareas del proyecto pero que, sin embargo, sirvieron para que se produjera una mayor negociación de significados (Bower y Kawaguchi, 2011; Sevilla Pavón, Martínez Sáez y Siqueira, 2011), un aprendizaje entre pares (Boud, Cohen y Sampson, 1999; Foster y Ohta, 2014), un uso auténtico de la lengua meta (Gilmore, 2007) y unas mayores necesidades de poner en práctica diferentes estrategias relacionadas con la competencia comunicativa intercultural (Byram, 1997; Koch, 2009; Wiseman, 2001). Todos los factores anteriormente señalados se consideran propulsores de un aprendizaje construido socialmente (Vygotsky, 1978) y más significativo (Mayer, 2002).

\subsection{Volumen de trabajo}

Además, algunos estudiantes manifestaron su opinión de que el volumen del trabajo era excesivo y de que la organización temporal, estructural y de trabajo se dificultaban a la hora de tener que coordinar horarios con estudiantes residentes en una zona horaria diferente, al otro lado del océano. Esos aspectos reflejan la importancia de la coordinación entre las docentes encargadas de los proyectos a la hora de confeccionar el cronograma de actividades. Asimismo, recibió críticas un aspecto que ha sido identificado por numerosos autores como determinante para el éxito de un intercambio de telecolaboración: la interdependencia positiva (Dooly, 2008; Fuchs, 2007; Guth y Helm, 2011; Yang, Huiju, Cen, y Huang, 2014). Así, la falta de respuesta por parte de algunos de los compañeros de telecolaboración asignados provocó sentimientos de frustración e impotencia en los estudiantes afectados, quienes se vieron perjudicados por el menor interés y compromiso de estos compañeros. Ello supuso un lastre en la realización del proyecto y amenazó con tener un impacto negativo en los resultados finales de los alumnos afectados. 
Las críticas anteriormente señaladas serán de utilidad de cara a mejorar futuras ediciones del proyecto, cuyas líneas prioritarias habrán de ser:

- el diseño de mecanismos que impidan la falta de reciprocidad y garanticen la interdependencia positiva entre las diferentes parejas y grupos de alumnos, para así vencer la consecuente frustración que dicha falta acarrearía;

- un mayor apoyo y coordinación entre el profesorado y alumnado de las instituciones participantes, que permita una realización correcta de las actividades y tareas en tiempo y forma, así como un mayor fomento de la participación y la interdependencia positiva entre los alumnos.

Estos y otros aspectos se tendrán en cuenta de cara al próximo curso académico, en que se espera seguir contando con el apoyo institucional adecuado para dar continuidad al proyecto y, de este modo, permitir que los alumnos se beneficien de la telecolaboración, que se perfila como una metodología de aprendizaje activa en que la comunicación intercultural desempeña un papel central que, en palabras de los estudiantes, les permite expandir sus horizontes al hacerse conscientes de que "el mundo puede ser tan distinto y similar al mismo tiempo".

\section{CONSIDERACIONES FINALES}

La inclusión del proyecto de telecolaboración aquí descrito como parte integral de la asignatura troncal "Inglés para los Negocios III" demuestra que en un número creciente de instituciones la "normalización" (Bax, 2000) de las TIC en las prácticas pedagógicas está pasando de ser una utopía a una realidad acorde con las necesidades, intereses y preferencias del alumnado del siglo XXI, considerados "nativos digitales" (Prensky, 2001). La familiarización con el uso de las TIC de estos "nativos digitales" les permite servirse de las mismas para llevar a cabo tareas y acceder a modalidades de aprendizaje que no serían posibles sin ellas, como es el caso del acceso a input lingüístico auténtico y variado o el acceso directo a la cultura meta por parte de los estudiantes que participen proyectos de telecolaboración como el que nos ocupa.

El apoyo institucional y la coordinación interinstitucional figuran entre los elementos esenciales en la puesta en práctica de un proyecto de telecolaboración y la realización exitosa del mismo. También cabe destacar la importancia de una buena planificación y de unos principios pedagógicos sólidos que guíen el uso de las TIC y su integración y normalización en el aula. Los resultados del estudio descrito en el presente artículo muestran alumnos responsables de su propio proceso de aprendizaje, críticos y conscientes de las ventajas y desafíos que conllevaban proyectos de tal magnitud, realizados en paralelo con estudiantes al otro lado del Atlántico, habiendo significativas diferencias culturales y lingüísticas, además de horarias, entre los participantes, las cuales se han de salvar mediante la comunicación intercultural efectiva y eficaz. 
Se espera que las críticas constructivas recibidas sirvan para mejorar el proyecto de cara a futuras ediciones del mismo. Además, entre las futuras líneas de investigación se incluye el diseño de un marco pedagógico que permita implementar el proyecto en otras áreas del conocimiento e idiomas. En el campo del inglés, además de aplicarse el proyecto a la enseñanza del inglés como lengua extranjera y del inglés con fines específicos en su vertiente de inglés para los negocios, también puede aplicarse en otras áreas, tanto dentro del campo de inglés para fines específicos (inglés para el área médica, inglés para estudios científicotécnicos e ingenierías) como en áreas más distantes, tales como la didáctica de la literatura. Ello serviría, por ejemplo, para llenar un hueco en la literatura, ya que son muy escasos - por no decir inexistentes- los estudios que se centran en la instrumentalización de la literatura a través de tareas de telecolaboración.

\section{BIBLIOGRAFÍA}

ÁLVAREZ GONZÁLEZ, Severina (2010): "La relevancia del enfoque intercultural en el aula de lengua extranjera", Madrid, Revista Nebrija de Lingüística Aplicada, $9,1-15$.

ANDERSON, L. W. y D. R. KRATHWOHL (eds., 2001). A taxonomy for learning, teaching, and assessing: A revision of Bloom's Taxonomy of Educational Objectives, New York, Longman.

BANDURA, Albert (2001): "The changing face of psychology at the dawning of a globalization era", Ottawa, Canadian Psychology, 42, 12-24.

BAX, Stephen (2000): "Putting technology in its place: ICT in modern foreign language". En FIELD, K. (ed.), Issues in modern foreign language teaching, Abingdon, Routledge/Falmer, 208-219.

BAX, Stephen (2003): "CALL: Past, present and future", Philadelphia, System, 31, 13-28.

BELZ, Julie Anne (2003): "Linguistic perspectives on the development of intercultural competence in telecollaboration", Language Learning \& Technology, 7 (2), 68-117. _http://ltt.msu.edu/vol7num2/belz/default.html [consulta: 23 febrero 2016].

Bloom, B. S., M. D. Engelhart, E. J. Furst, W. H. HiLl y D. R. KRATHWOHL (1956): Taxonomy of educational objectives: The classification of educational goals. Handbook I: Cognitive domain, New York, David McKay Company.

Boud, D., R. COHEN y J. SAMPSON (1999): "Peer Learning and Assessment", Assessment \& Evaluation in Higher Education, Milton Park, Abingdon-onThames, Taylor \& Francis, 24, (4), 413-426.

BOWER, J. y K. SATOMI (2011): "Negotiation of meaning and corrective feedback in Japanese/English eTandem", Language Learning \& Technology, 15 (1): 4171. http://ltt.msu.edu/issues/february2011/bowerkawaguchi.pdf [consulta: 24 febrero 2016]. 
BRISLIN, R. W. y T. YoshidA (1994): Intercultural communication training: An introduction, Thousand Oaks, Sage.

BYRAM, Michael (1997): Teaching and assessing intercultural communicative competence, Clevendon, Multilingual Matters.

BYRAM, Michael (2000): "Assessing Intercultural Competence in Language Teaching”, Aarhus, Sprogforum, 18 (6), 8-13.

BYRAM, M. y M. FLEMING (1998): Language learning in intercultural perspective, Cambridge, Cambridge University Press.

ChAMBers, A. y S. BAX (2006): "Making CALL work: Towards normalization", Philadelphia, System, 34, 465-479.

Comisión Buropea. Boletín de noticias. http://ec.europa.eu/education/opportunities/school/cooperation-schools_en.htm [consulta: 16 febrero 2016].

Consejo de Europa (2001): Common European framework of reference for languages: Learning, teaching and assessment, Cambridge, Cambridge University Press.

Dooly, Melinda (2008): "Constructing knowledge together", en M. Dooly (ed.), Telecollaborative language learning. A guidebook to moderating intercultural collaboration online, Bern/Wien, Peter Lang, 21-44.

DoOLY, M. y R. O'DowD (2012): Researching online interaction and exchange in foreign language education, Bern/Wien, Peter Lang.

ECK, A., L. LEGENHAUSEN y D. WOLFF (1995): Telekommunikation und Fremdsprachenunte-rricht: Informationen, Projekte, Ergebnisse, Bochum, Broschiert.

Foster, P. y A. S. OHTA (2005): "Negotiation for Meaning and Peer Assistance in Second Language Classrooms", Applied Linguistics, Oxford, Oxford Journals, 26 (3), 402-430.

FuCHS, Carolin (2007): "Student language teachers as intercultural learners in CMC-based project work", Göteburg, Journal of Intercultural Communication, 13. http://www.immi.se/intercultural/nr13/fuchs.htm [consulta: 23 junio 2016].

GIDDENS, Anthony (2014): Sociología, Madrid, Alianza.

GILMORE, Alex (2007): Authentic materials and authenticity in foreign language learning. Language Teaching, Cambridge, Cambridge Journals, 97-118.

GuILHERME, Manuela (2004): "Intercultural Competence", en BYRAM, M. (ed.): Routledge encyclopaedia of language teaching and learning, London, Routledge.

GUTH, S. y F. HELM (2011): Developing multiliteracies in ELT through telecollaboration, Oxford, Oxford University Press. ELT Journal.

GUTH, S. y F. HELM (2012): "Developing multiliteracies in ELT through telecollaboration", English Language Teaching Journal, Oxford, Oxford Journals, 66 (1). 42-51. 
Guth, S., F. Helm y R. O’DowD (2014): "Telecollaborative foreign language networks in European universities: A report on current attitudes and practices", Bellaterra Journal of Teaching \& Learning Language \& Literature, Barcelona, $\mathrm{UAB}, 7$ (4), 1-14.

HAUCK, M. y B. YOUNGS (2008): "Telecollaboration in multimodal environments: The impact on task design and learner interaction", Computer Assisted Language Learning, Milton Park, Abingdon-on-Thames, Taylor \& Francis, 21 (2), 87-124.

JONES, Leo (2007): The student-centered classroom, Cambridge, Cambridge University Press.

Kern, R., P. WARe y M. WARSchauer (2004): "Crossing frontiers: New directions in online pedagogy and research", Annual Review of Applied Linguistics, Cambridge, Cambridge Journals, 24, 243-260.

KNOBEL, M. y C. LANKSHEAR (2008): "Digital literacy and participation in online social networking spaces", en LANKSHEAR, C. and KNOBEL, M. (eds.), Digital Literacies, New York, Peter Lang, 249-278.

$\mathrm{KoCH}$, Gertraud (2009): "Intercultural Communication and Competence Research through the Lens of an Anthropology of Knowledge", Forum Qualitative Sozialforschung / Forum: Qualitative Social Research, 10 (1), Art. 15, 1-21.

LiTTLE, David (1997): "Learner autonomy in the foreign language classroom: theoretical foundations and some essentials of pedagogical practice", Zeitschrift für Fremdsprachenforschung, Uhr, Oldenburg Schulbuchverlag, 8 (2), 227-244.

LIZCANO, Emmánuel (1999): "La metáfora como analizador social", Madrid, EMPIRIA, Revista de Metodología de Ciencias Sociales, 2, 29-60.

MARTIN, A. y D. MADIGAN (2006): Digital Literacies for Learning, London, Facet Publishing.

MAYER, Richard (2002): "Rote Versus Meaningful Learning", Special Issue: Revising Bloom's Taxonomy, Milton Park, Abingdon-on-Thames, Taylor \& Francis, 41 (4), 226-232.

MEYER, Meinert (1990). "Developing transcultural competence: case studies of advanced foreign langauge learners", en ButTJES, D. y BYRAM, M. (eds.): Mediating languages and cultures: Towards an intercultural theory of foreign language education, Clevedon, Multilingual Matters.

O'DOWD, Robert (2013): The competences of the telecollaborative teacher. The
Language
Learning
Journal,
43
(2),
194-207.

http://dx.doi.org/10.1080/09571736.2013.853374 [consulta: 14 junio 2016].

PASTOR, Susana (2005): "La enseñanza de segundas lenguas", en LÓPEZ, A. y Gallardo, B. (eds.) Conocimiento y Lenguaje, Valencia, PUV.

PÉREZ CAÑADO, María Luisa (2010): "Globalisation in foreign language teaching: Establishing transatlantic links in higher education". Higher Education Quarterly, 64: 392-412. http://dx.doi.org/doi: 10.1111/j.14682273.2010.00451.x [consulta: 14 junio 2016]. 
PÉREZ CAÑADO, María Luisa (2013, ed.): Competency-based language teaching in higher Education, Dordrecht, Springer.

PRENSKY, M. (2001): "Digital Natives, Digital Immigrants Part 1", On the Horizon, 9 (5): 1-6._http://dx.doi.org/10.1108/10748120110424816 [consulta: 16 febrero 2016].

RICHARDS, J. y T. RODGERS (2014): Approaches and methods in language teaching, Cambridge, Cambridge University Press.

Sevilla-Pavón, A, A. Martínez-SÁez y J. M. De Siqueira (2011): "Selfassessment and tutor assessment in online language learning materials: InGenio FCE online course and tester", en THOUESNY, S. y BRADLEY, L. (eds.) Second language teaching and learning with technology: Views of emergent researchers, Dublin, Research-publishing.net http://dx.doi.org/10.14705/rpnet.2011.000006 [consulta: 24 febrero 2016].

SEVILla PAVÓN, A. y J. HABA OSCA (En prensa): "Learning from real life and not books: A gamified approach to task design in transatlantic telecollaboration", Ibérica, 32, Castellón, AELFE.

SKeHAN, Peter (2003a): "Focus on form, tasks, and technology", Computer Assisted Language Learning, Milton Park, Abingdon-on-Thames, Taylor \& Francis, 16 (5), 391-411.

SKeHAN, Peter (2003b): “Task-based instruction”, Language Teaching, Cambridge, Cambridge Journals, 36, 1-34.

VAN DIJK, Jan (2005): The deepening divide, inequality in the information society, Thousand Oaks/London/New Delhi, Sage Publications.

VAN DIJK, J. y K. HACKER (2003): "The digital divide as a complex and dynamic phenomenon", The Information Society, Milton Park, Abingdon-on-Thames, Taylor \& Francis, 19 (4), 315-326.

VINAGRE LARANJEIRA, Margarita (2014): "El desarrollo de la competencia intercultural en los intercambios telecolaborativos". RED - Revista de Educación a Distancia, 41, 1-22.

VINAGRE LARANJEIRA, Margarita (2016): "Training teachers for virtual collaboration: A case study". British Journal of Educational Technology, 47 (4), 787-802.

VyGOTSKY, Lev (1978): Mind and society. Cambridge, Harvard University Press.

WILLIS, Jane (1996): A framework for task-based learning. Longman handbooks for language teachers, Harlow, Longman.

WISEMAN, Richard (2001): "Intercultural Communication Competence", en GUDYKUNST, W. y MODY, B. (eds.), Handbook of intercultural and international communication, Newbury Park, Sage Publications.

YANG, J., Y. HUIJI, S.J. CEN, y R. HUANG (2014): "Strategies for smooth and effective cross-cultural online collaborative learning", Alberta, Educational Technology \& Society, 17 (3), 208-221. 